\title{
Impact of endoscopic versus open saphenous vein harvest technique on late coronary artery bypass grafting patient outcomes in the ROOBY (Randomized On/Off Bypass) Trial
}

Marco A. Zenati, MD, ${ }^{\mathrm{a}, \mathrm{b}}$ A. Laurie Shroyer, PhD, ${ }^{\mathrm{c}, \mathrm{d}}$ Joseph F. Collins, ScD, ${ }^{\mathrm{e}}$ Brack Hattler, MD, ${ }^{\mathrm{c}}$ Takeyoshi Ota, MD, PhD, ${ }^{\mathrm{f}, \mathrm{g}}$ G. Hossein Almassi, MD, ${ }^{\mathrm{h}}$ Morteza Amidi, MD, ${ }^{\mathrm{f}}$ Dimitri Novitzky, MD, PhD, ${ }^{\mathrm{i}}$ Frederick L. Grover, $\mathrm{MD},{ }^{\mathrm{c}}$ and Ali F. Sonel, $\mathrm{MD}^{\mathrm{f}}$

Objective: In the Randomized On/Off Bypass (ROOBY) Trial, the efficacy of on-pump versus off-pump coronary artery bypass grafting was evaluated. This ROOBY Trial planned subanalysis compared the effects on postbypass patient clinical outcomes and graft patency of endoscopic vein harvesting and open vein harvesting.

Methods: From April 2003 to April 2007, the technique used for saphenous vein graft harvesting was recorded in 1471 cases. Of these, 894 patients (341 endoscopic harvest and 553 open harvest) also underwent coronary angiography 1 year after coronary artery bypass grafting. Univariate and multivariable analyses were used to compare patient outcomes in the endoscopic and open groups.

Results: Preoperative patient characteristics were statistically similar between the endoscopic and open groups. Endoscopic vein harvest was used in $38 \%$ of the cases. There were no significant differences in both short-term and 1-year composite outcomes between the endoscopic and open groups. For patients with 1-year catheterization follow-up $(\mathrm{n}=894)$, the saphenous vein graft patency rate for the endoscopic group was lower than that in the open harvest group $(74.5 \%$ vs $85.2 \%, P<.0001)$, and the repeat revascularization rate was significantly higher $(6.7 \%$ vs $3.4 \%, P<.05)$. Multivariable regression documented no interaction effect between endoscopic approach and off-pump treatment.

Conclusions: In the ROOBY Trial, endoscopic vein harvest was associated with lower 1-year saphenous vein graft patency and higher 1-year revascularization rates, independent of the use of off-pump or on-pump cardiac surgical approach. (J Thorac Cardiovasc Surg 2011;141:338-44)

Earn CME credits at

http://cme.ctsnetjournals.org

From the Veterans Affairs Boston Healthcare System, ${ }^{\text {a }}$ West Roxbury, Mass; the Division of Cardiac Surgery, ${ }^{\text {b }}$ Brigham \& Women's Hospital, Harvard Medical School, Boston, Mass; Eastern Colorado Healthcare System, ${ }^{\mathrm{C}}$ Department of Veterans Affairs, Denver, Colo; Northport Veterans Affairs Medical Center, ${ }^{\mathrm{d}}$ Northport, NY; Cooperative Studies Program Coordinating Center, ${ }^{\mathrm{e}}$ Veterans Affairs Medical Center, Perry Point, Md; Veterans Affairs Pittsburgh Healthcare System, ${ }^{\mathrm{f}}$ Pittsburgh, Pa; Heart, Lung and Esophageal Surgery Institute, ${ }^{\mathrm{g}}$ University of Pittsburgh Medical Center, Pittsburgh, Pa; Medical College of Wisconsin and Zablocki Veterans Affairs Medical Center, ${ }^{\mathrm{h}}$ Milwaukee, Wis; and the Department of Surgery, ${ }^{\mathrm{i}}$ University of South Florida, Tampa, Fla.

Supported by the Cooperative Studies Program of the Department of Veterans Affairs, Office of Research and Development, and by the Department of Veterans Affairs Central Office, Office of Patient Care Services, Washington, DC.

Disclosures: Authors have nothing to disclose with regard to commercial support.

Read at the 90th Annual Meeting of the American Association for Thoracic Surgery, Toronto, Ontario, Canada, May 1-5, 2010.

Received for publication April 30, 2010; revisions received Sept 27, 2010; accepted for publication Oct 1, 2010; available ahead of print Dec 6, 2010.

Address for reprints: Marco A. Zenati, MD, Division of Cardiac Surgery, BHS, 1400 VFW Pkwy (112), West Roxbury, MA 02132 (E-mail: Marco_Zenati@hms. harvard.edu).

0022-5223/\$0.00

Published by Elsevier Inc. on behalf of The American Association for Thoracic Surgery

doi:10.1016/j.jtcvs.2010.10.004
Since the publication of the first clinical report on endoscopic greater saphenous vein (SVG) graft harvesting $(\mathrm{EVH})$ in $1996,{ }^{1} \mathrm{EVH}$ has increased in popularity to become the preferred method of SVG harvesting in the United States and is now a class IIa recommendation on the basis of level B evidence. ${ }^{2}$ According to the Society of Thoracic Surgeons' National Database (www.sts.org), EVH was used in $70 \%$ of coronary artery bypass grafting (CABG) procedures in 2008.

A significant number of studies have demonstrated shortterm advantages of EVH, including less wound morbidity, less pain, better cosmetic results, and improved patient satisfaction relative to open greater SVG harvesting $(\mathrm{OVH}) .^{3-7}$ More recently, long-term follow up of the Project of Ex-vivo Vein Graft Engineering via Transfection IV trial (PREVENT IV) showed worse outcomes with EVH. ${ }^{8,9}$ The rate of SVG failure was significantly higher among those who underwent EVH (46.7\% vs $38.0 \%$; odds ratio, $1.45 ; 95 \%$ confidence interval, 1.20-1.76). At 3 years, EVH was also associated with a significantly higher combined rate of death, myocardial infarction, or repeat revascularization $(20.2 \%$ vs $17.4 \%$; adjusted hazard ratio, $1.22 ; 95 \%$ confidence interval, 1.01-1.47). Because of the potential implications of the longer term impact on outcomes, the role of EVH is currently the object of much controversy in the literature. ${ }^{10-12}$ 


$\begin{array}{ll}\begin{array}{ll}\text { Abbreviations and Acronyms } \\ \text { sCABG }\end{array} & \text { coronary artery bypass grafting } \\ \text { EPIC } & =\text { Evaluation of the PAS-Port in } \\ & \text { Coronary Surgery [trial] } \\ \text { EVH } & \text { endoscopic greater saphenous vein } \\ & \text { graft harvest } \\ = & \text { open greater saphenous vein graft } \\ \text { OVH } & \text { harvest } \\ & \text { PREVENT = Project of Ex-vivo Vein Graft } \\ \text { IV } & \text { Engineering via Transfection IV } \\ & \text { [trial] } \\ \text { ROOBY }= & \text { Randomized On/Off Bypass [trial] } \\ \text { SVG } & =\text { saphenous vein graft } \\ \text { VA } & =\text { Veterans Affairs }\end{array}$

The results of the Randomized On/Off Bypass (ROOBY) Trial were recently published. ${ }^{13}$ This planned subanalysis of the ROOBY Trial examines clinical outcomes in ROOBY Trial patients who underwent SVG harvest by endoscopic and open techniques. Specifically, the ROOBY trial's primary short-term and 1-year clinical composite outcomes and graft patency are compared between patients who underwent EVH and those who underwent OVH.

\section{MATERIALS AND METHODS \\ Study Design}

The ROOBY Trial was a randomized, controlled, single-blind study that randomly allocated patients undergoing nonemergency CABG to off-pump versus on-pump techniques at 18 participating Veterans Affairs (VA) Medical Centers in the United States (ClinicalTrials.gov Identifier: NCT00032630). The ROOBY study protocol, including this analysis, was approved by each participating VA Medical Center's institutional review board and research and development office. Informed consent was obtained for each patient.

Details of the ROOBY study design and inclusion and exclusion criteria have been published previously. ${ }^{14}$ At the beginning of the study in 2002, the technique used to harvest SVG was not recorded on the study forms. In 2003, in light of emerging interest with EVH, institutional review board approval was obtained at every site to revise the study forms to allow collection of this data. Surgeons were encouraged to use whichever harvesting technique they preferred in a balanced fashion between off- and onpump arms.

\section{Study End Points}

The ROOBY Trial's primary short-term end point was a composite of death or major perioperative complication (reoperation, new mechanical support, cardiac arrest, coma, stroke, or renal failure requiring dialysis) occurring within 30 days after surgery or before hospital discharge, whichever was later. The 1-year composite outcome was composed of all-cause mortality, repeat revascularization between 30 days to 1 year, or nonfatal myocardial infarction between 30 days and 1 year. Graft patency was determined by 1-year follow-up cardiac catheterizations overread by an angiographic core laboratory that was blinded to all treatment details (including EVH vs OVH). FitzGibbon grading was used to score graft patency. ${ }^{15}$ For this analysis, overall graft patency (open vs occluded) and the proportion of patients with at least 1 occluded SVG were evaluated.

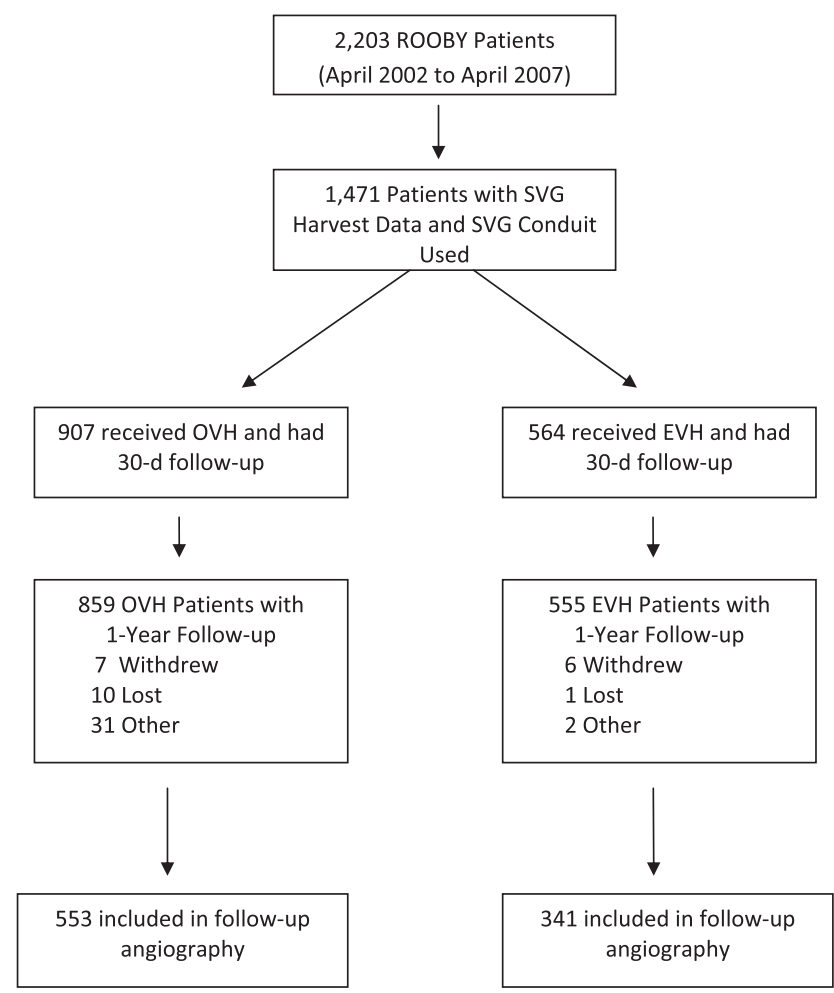

FIGURE 1. Enrollment, treatment, and follow-up. ROOBY, Randomized On/Off Bypass Trial; $S V G$, saphenous vein graft; $O V H$, open greater saphenous vein graft harvest; $E V H$, endoscopic greater saphenous vein graft harvest.

\section{Surgical Procedure}

All CABG procedures were performed through a standard median sternotomy. The 18 ROOBY Trial VA Medical Centers represented in this substudy were as follows: Albuquerque, Asheville, Cleveland, Dallas, Denver, Durham, Gainesville, Los Angeles, Miami, Milwaukee, New York, Palo Alto, Pittsburgh, Portland, San Antonio, San Francisco, Tampa, and Washington. EVH was not performed at 5 centers. Within the VA Medical Centers that used $\mathrm{EVH}$, the use rate (determined for the cases performed with at least $1 \mathrm{SVG}$ conduit placed) ranged from $1.5 \%$ to $100 \%$. The quality of the SVG was evaluated intraoperatively and graded as good, intermediate, or poor by the surgeon.

\section{Statistical Analysis}

Continuous variables were reported as mean $\pm \mathrm{SD}$, and categoric variables were reported as frequencies. For categoric variables, a 2-tailed continuity-corrected $\chi^{2}$ or Fisher's Exact test was used for univariate analysis. The Student $t$ test or the Mann-Whitney U test was used for continuous variables according to distribution. Multivariable logistic regression models were built to predict the dichotomous substudy outcomes of interest. For these, the variables considered eligible for model entry included the 30-day operative mortality risk estimate (as a summary patient risk measure), an EVH indicator variable, an off-pump indicator variable, and an interaction term for the combination of EVH and off-pump techniques. A sensitivity analysis was performed to evaluate for a differential EVH versus OVH impact for high-volume versus low-volume EVH centers.

\section{RESULTS}

From February 2002 through April 2007, the ROOBY Trial enrolled 2203 patients. Beginning in April 2003, 
TABLE 1. Preoperative characteristics for all patients $(n=1471)$

\begin{tabular}{lccc}
\hline & $\begin{array}{c}\text { Endoscopic } \\
(\mathbf{n}=\mathbf{5 6 4})\end{array}$ & $\begin{array}{c}\text { Open } \\
(\mathbf{n}=\mathbf{9 0 7})\end{array}$ & $\boldsymbol{P}$ value \\
\hline Age $(\mathrm{y}$, mean $\pm \mathrm{SD})$ & $63.0 \pm 8.0$ & $62.6 \pm 8.4$ & .28 \\
Male & $563(99.8 \%)$ & $897(98.9 \%)$ & .06 \\
Chronic obstructive & $138(24.5 \%)$ & $168(18.5 \%)$ & .007 \\
$\quad$ pulmonary disease & & & \\
Cerebrovascular accident & $49(8.7 \%)$ & $62(6.8 \%)$ & .22 \\
Peripheral vascular disease & $84(14.9 \%)$ & $129(14.2 \%)$ & .76 \\
Diabetes & $256(45.4 \%)$ & $408(45.0 \%)$ & .91 \\
Hypertension & $490(86.9 \%)$ & $801(88.3 \%)$ & .41 \\
Hyperlipidemia & $500(89.0 \%)$ & $791(87.4 \%)$ & .41 \\
Renal insufficiency & $40(7.1 \%)$ & $80(8.8 \%)$ & .28 \\
$\quad($ creatinine $>1.5$ mg/dL) & & & \\
Left ventricular ejection fraction & & & \\
$\quad<35 \%$ & $30(5.5 \%)$ & $51(5.7 \%)$ & .50 \\
$35 \%-44 \%$ & $58(10.7 \%)$ & $113(12.7 \%)$ & \\
$\quad>44 \%$ & $455(83.8 \%)$ & $723(81.5 \%)$ & \\
Urgent status & $81(14.4 \%)$ & $110(12.1 \%)$ & .23 \\
No. of diseased vessels & & & \\
$\quad 1$ & $20(3.6 \%)$ & $39(4.3 \%)$ & .44 \\
2 & $157(25.3 \%)$ & $228(25.3 \%)$ & \\
3 & $383(68.4 \%)$ & $634(70.4 \%)$ & \\
On-pump versus off-pump & $285: 279$ & $449: 458$ & $>.999$ \\
\hline
\end{tabular}

Data represent numbers of patients except as marked.

prospective collection of data regarding SVG harvest technique was begun (Figure 1). A total of 1471 patients (564 EVH, 907 OVH) with SVG used as a bypass conduit had the harvesting technique used recorded The 30-day composite end point was known in all cases. One-year composite follow-up was determined for $96 \%$ of these patients (555 $\mathrm{EVH}, 859 \mathrm{OVH})$. Follow-up angiography was obtained for 894 subjects (341 EVH, $553 \mathrm{OVH}$ ).

For both the population of all patients with SVG harvest approach recorded $(\mathrm{n}=1471)$ and the subset with 1-year cardiac catheterization $(n=894)$, the preoperative patient characteristics were generally balanced between the EVH and the OVH groups (Tables 1 and 2). Almost all patients were male $(99 \%)$, reflective of the VA cardiac surgical patient population. Sixty-eight percent of patients had 3-vessel coronary artery disease, and approximately $83 \%$ of patients had preserved left ventricular function. Fewer than $15 \%$ of study patients required urgent surgery.

The quality of the harvested SVG was assessed as good in $81.6 \%$ of EVH and $85.6 \%$ of OVH patients, intermediate in $15.7 \%$ of EVH and $12.7 \%$ of OVH patients, and poor in $2.8 \%$ of EVH and $1.7 \%$ of $\mathrm{OVH}$ patients (difference not significant).

For the short-term composite end point, there was no significant difference between EVH and OVH groups (Tables 3 and 4). More OVH patients than EVH patients $(1.3 \%)$ had renal failure $(1.3 \%$ vs $0.0 \%, P=.01)$, and more $\mathrm{OVH}$ patients needed new mechanical support $(1.7 \%$ vs $0.4 \%$, $P=.02)$.
TABLE 2. Preoperative characteristics for patients with 1-year catheterization $(\mathbf{n}=894)$

\begin{tabular}{|c|c|c|c|}
\hline & $\begin{array}{c}\text { Endoscopic } \\
(n=341)\end{array}$ & $\begin{array}{c}\text { Open } \\
(\mathbf{n}=\mathbf{5 5 3})\end{array}$ & $P$ value \\
\hline Age $(y$, mean \pm SD $)$ & $62.2 \pm 7.5$ & $61.9 \pm 7.9$ & .53 \\
\hline Male & $341(100 \%)$ & $546(98.7 \%)$ & .05 \\
\hline $\begin{array}{l}\text { Chronic obstructive } \\
\text { pulmonary disease }\end{array}$ & $75(22.0 \%)$ & $97(17.5 \%)$ & .12 \\
\hline Cerebrovascular accident & $22(6.5 \%)$ & $31(5.6 \%)$ & .66 \\
\hline Peripheral vascular disease & $50(14.7 \%)$ & $64(11.6 \%)$ & .18 \\
\hline Diabetes & $150(44.0 \%)$ & $239(43.2 \%)$ & .84 \\
\hline Hypertension & $293(85.9 \%)$ & $477(86.3 \%)$ & .92 \\
\hline Hyperlipidemia & $303(89.4 \%)$ & $479(86.8 \%)$ & .29 \\
\hline $\begin{array}{l}\text { Renal insufficiency } \\
\quad(\text { creatinine }>1.5 \mathrm{mg} / \mathrm{dL})\end{array}$ & $13(3.8 \%)$ & $31(5.6 \%)$ & .27 \\
\hline \multicolumn{4}{|c|}{ Left ventricular ejection fraction } \\
\hline$<35 \%$ & $16(4.8 \%)$ & $24(4.4 \%)$ & .91 \\
\hline $35 \%-44 \%$ & $34(10.2 \%)$ & $59(10.9 \%)$ & \\
\hline$>44 \%$ & $283(85.0 \%)$ & $459(84.7 \%)$ & \\
\hline Unknown & $8(2.3 \%)$ & $11(2.0 \%)$ & \\
\hline Urgent status & $52(15.2 \%)$ & $71(12.8 \%)$ & .32 \\
\hline \multicolumn{4}{|l|}{ No. of diseased vessels } \\
\hline 1 & $16(4.7 \%)$ & $25(4.5 \%)$ & .60 \\
\hline 2 & $99(29.2 \%)$ & $145(26.2 \%)$ & \\
\hline 3 & $224(66.1 \%)$ & $383(69.3 \%)$ & \\
\hline On-pump versus off-pump & $166: 175$ & $269: 284$ & $>.999$ \\
\hline
\end{tabular}

Regarding the 1-year composite outcome, there were no differences between EVH and OVH groups (Tables 5 and 6). For the subgroup of patients with 1-year cardiac catheterization studies, the rate repeat revascularization was significantly higher in the EVH group than in the OVH group $(6.7 \%$ vs $3.4 \%, P<.05)$, whereas the rates of nonfatal myocardial infarction and of death were similar (Table 6). Only 2 of the 42 patients that needed repeat revascularization $(4.8 \%)$ required reoperative CABG, whereas the vast majority $(40 / 42,95.2 \%)$ required percutaneous coronary intervention.

Graft patency results in the subgroup of 894 patients with follow-up angiography are detailed in Table 7 . A mean of

TABLE 3. Thirty-day outcomes for all patients $(n=1471)$

\begin{tabular}{lccc}
\hline & $\begin{array}{c}\text { Endoscopic } \\
(\mathbf{n}=\mathbf{5 6 4})\end{array}$ & $\begin{array}{c}\text { Open } \\
(\mathbf{n}=\mathbf{9 0 7})\end{array}$ & $\boldsymbol{P}$ value \\
\hline 30-d Mortality & $2(0.4 \%)$ & $10(1.1 \%)$ & .15 \\
Reoperation for bleeding & $14(2.5 \%)$ & $22(2.4 \%)$ & $>.999$ \\
Repeat bypass & $3(0.5 \%)$ & $13(1.4 \%)$ & .13 \\
Cardiac arrest & $8(1.4 \%)$ & $13(1.4 \%)$ & $>.999$ \\
Renal failure & $0(0.0 \%)$ & $12(1.3 \%)$ & .005 \\
Stroke & $4(0.7 \%)$ & $10(1.1 \%)$ & .59 \\
New mechanical support & $2(0.4 \%)$ & $15(1.7 \%)$ & .02 \\
30-d Composite end point* & $26(4.6 \%)$ & $64(7.1 \%)$ & .06 \\
\hline Da
\end{tabular}

Data represent numbers of patients. *Primary 30-day composite end point was death, reoperation, repeat bypass, cardiac arrest, stroke, new mechanical support, or renal failure requiring dialysis. 
TABLE 4. Thirty-day outcomes for patients with 1-year catheterization $(n=894)$

\begin{tabular}{lccc}
\hline & $\begin{array}{c}\text { Endoscopic } \\
(\mathbf{n}=\mathbf{3 4 1})\end{array}$ & $\begin{array}{c}\text { Open } \\
(\mathbf{n}=\mathbf{5 5 3})\end{array}$ & $\boldsymbol{P}$ value \\
\hline 30-d Mortality & 0 & 0 & NA \\
Reoperation for bleeding & $9(2.6 \%)$ & $9(1.6 \%)$ & .33 \\
Repeat bypass & $1(0.3 \%)$ & $2(0.4 \%)$ & $>.999$ \\
Cardiac arrest & $4(1.2 \%)$ & $4(0.7 \%)$ & .49 \\
Renal failure & $0(0.0 \%)$ & $3(0.5 \%)$ & .29 \\
Stroke & $1(0.3 \%)$ & $4(0.7 \%)$ & .65 \\
New mechanical support & $0(0 \%)$ & $4(0.7 \%)$ & .30 \\
30-d Composite end point* & $12(3.5 \%)$ & $24(4.3 \%)$ & .60 \\
\hline
\end{tabular}

Data represent numbers of patients. $N A$, Not applicable. *Primary 30-day composite end point was death, reoperation, repeat bypass, cardiac arrest, stroke, new mechanical support, or renal failure requiring dialysis.

2.02 SVGs were placed per patient, and a total of 1807 SVGs were assessed for patency. The incidence of a patient having 1 or more occluded SVGs on follow-up angiography was $41.3 \%$ in the EVH group, compared with $28.0 \%$ in the OVH group $(P<.0001)$. Overall SVG patency in the EVH group was $74.5 \%$, which was significantly worse than the $85.2 \%$ rate in the OVH group $(P<.0001)$.

According to multivariable regression analysis, EVH was not found to be an independent and statistically significant predictor of the 1-year composite outcome when other factors were held constant. Further, no interaction was found between the SVG harvesting technique (EVH vs $\mathrm{OVH})$ and the use of an on-pump versus off-pump approach (difference not significant). Additionally, sensitivity analysis found no differential $\mathrm{EVH}$ versus $\mathrm{OVH}$ effect for highvolume versus low-volume EVH centers.

\section{DISCUSSION}

Although it has been established that internal thoracic arteries have clinical and patency advantages relative to SVGs, ${ }^{16}$ SVG conduits are still commonly used during CABG. EVH technique has been developed in an effort to decrease the complications associated with $\mathrm{OVH}$ and has been documented to reduce wound-related morbidity. ${ }^{6,7}$

For patients in the ROOBY Trial with EVH versus OVH data captured, there were no major differences found in the ROOBY Trial's primary short-term and 1-year composite end points. Relative to $\mathrm{OVH}, \mathrm{EVH}$ was associated with

TABLE 5. One-year outcomes for all patients $(n=1414)$

\begin{tabular}{lccc}
\hline \multicolumn{1}{c}{ Late outcomes } & $\begin{array}{c}\text { Endoscopic } \\
(\mathbf{n}=\mathbf{5 5 5})\end{array}$ & $\begin{array}{c}\text { Open } \\
(\mathbf{n = 8 5 9 )}\end{array}$ & $\boldsymbol{P}$ value \\
\hline Revascularization & $29(5.2 \%)$ & $30(3.5 \%)$ & .13 \\
Myocardial infarction & $9(1.6 \%)$ & $15(1.7 \%)$ & $>.999$ \\
Death & $13(2.3 \%)$ & $25(2.9 \%)$ & .61 \\
1-y Composite end point* & $47(8.5 \%)$ & $62(7.2 \%)$ & .42 \\
\hline
\end{tabular}

Data represent numbers of patients. *Primary 1-year composite end point was death from any cause within 1 year after surgery, myocardial infarction between 30 days and 1 year, or any revascularization procedure between 30 days and 1 year.
TABLE 6. One-year outcomes for patients with 1-year catheterization $(\mathbf{n}=\mathbf{8 9 4})$

\begin{tabular}{lccr}
\hline \multicolumn{1}{c}{ Late outcomes } & $\begin{array}{c}\text { Endoscopic } \\
(\mathbf{n}=\mathbf{3 4 1})\end{array}$ & $\begin{array}{c}\text { Open } \\
(\mathbf{n}=\mathbf{5 5 3})\end{array}$ & $\boldsymbol{P}$ value \\
\hline Revascularization & $23(6.7 \%)$ & $19(3.4 \%)$ & .033 \\
Myocardial infarction & $6(1.8 \%)$ & $11(2.0 \%)$ & $>.999$ \\
Death & $1(0.3 \%)$ & $2(0.4 \%)$ & $>.999$ \\
1-y Composite end point* & $28(8.2 \%)$ & $27(4.9 \%)$ & .061 \\
\hline Data represent numbers of patients. *Primary 1-year composite end point was death \\
from any cause within 1 year after surgery, myocardial infarction between 30 days and \\
1 year, or any revascularization procedure between 30 days and 1 year.
\end{tabular}

poorer SVG patency and more frequent repeat revascularization. According to the sensitivity analysis performed, moreover, this EVH versus OVH effect was not different in the subgroup of high-volume EVH centers.

SVG patency was significantly lower in the EVH group than in the OVH group for both off-pump and on-pump CABG (data not shown). By multivariable analysis, the effect of EVH relative to OVH on graft patency appeared to be independent of the effects of off-pump versus on-pump approach (that is, no greater combined effect observed for the use of off-pump with EVH technique than seen when evaluating these effects in isolation). In addition, the higher repeat revascularization rate in the group of EVH patients with 1-year catheterization data may be explained by the higher rate of SVG occlusion driving percutaneous interventions at the time of angiography (Figure 2).

$\mathrm{EVH}$ commonly requires carbon dioxide to insufflate the subcutaneous cavity and bipolar cautery in the vicinity of SVGs, whereas neither insufflation nor bipolar energy is required for OVH. The use of cautery has been proposed to cause thermal injury to the vessel wall, which may impair the graft quality by compromising the viability of endothelial cells, resulting in platelet aggregation and thrombosis. Relatively longer manipulation times and the use of rigid devices in EVH may also cause direct mechanical injury to the SVGs. No study has addressed the effect of the use of carbon dioxide insufflation (either carbon dioxide itself or its gas pressure) on the quality of SVGs. Rousou and colleagues ${ }^{17}$ recently reported that relative to OVH, EVH adversely affects vein endothelial function. This finding is potentially important because endothelial dysfunction would adversely affect the entire vein graft, whereas heat or mechanical injuries would likely be localized.

It is also important to take into consideration the flowdynamic physics for graft patency. Disease severity in the distal coronary artery, so-called runoff, is significantly associated with graft patency. Specifically, poor runoff is associated with a high rate of graft occlusion. ${ }^{6,18}$ This factor was not assessed in our study.

Our study has additional limitations. Because the vast majority of ROOBY Trial patients were male, the conclusions may not be applicable to female patients, although 
TABLE 7. Patency outcomes $(n=894)$

\begin{tabular}{|c|c|c|c|c|c|}
\hline & Endoscopic (no.) & Open (no.) & Absolute percentage difference $(95 \%$ CI $)$ & Relative risk $(95 \%$ CI $)$ & $P$ value \\
\hline Occluded SVG $(\geq 1)$ & $141 / 341(41.3 \%)$ & $155 / 553(28.0 \%)$ & $13.3(6.9-19.8)$ & $1.23(1.11-1.36)$ & $<.0001$ \\
\hline SVG patency* & $513 / 689(74.5 \%)$ & $952 / 1118(85.2 \%)$ & $10.7(6.8-14.6)$ & $1.14(1.09-1.20)$ & $<.0001$ \\
\hline
\end{tabular}

$C I$, Confidence interval; $S V G$, saphenous graft. *FitzGibbon grade A or B.

female sex has not to our knowledge been identified as an independent risk factor for graft failure. In addition, this study did not randomize the assignment to the $\mathrm{EVH}$ or the OVH technique. Confounders related to surgeon's decision to use an EVH approach for SVG harvest may therefore have affected this study's findings.

As a potential positive attribute, this ROOBY Trial substudy was a multicenter study with many surgeons and midlevel providers with variable degrees of expertise. Thus the results may be more representative of EVH practice than single-center, "expert surgeon" or "expert midlevel provider" studies.

Although a few randomized studies comparing EVH and OVH have been published, they suffer from low volumes of patients, lack of angiographic assessment, or short followup. $^{7,19,20}$ In the EPIC (Evaluation of the PAS-Port in Coronary Surgery) Trial, worse SVG angiographic patency at 9 months was seen with EVH than with OVH $(79.2 \%$ vs $90.8 \%) .{ }^{21} \mathrm{~A}$ recent meta-analysis of 102 studies comparing the EVH and OVH in CABG concluded that long-term graft patencies in SVGs harvested by OVH were better than in those harvested by EVH (pooled odds ratio 1.25, $P=.0039) .{ }^{6}$ Our ROOBY Trial substudy supports the findings from this meta-analysis, because the 1-year patencies of SVGs harvested with EVH were lower than seen with $\mathrm{OVH}$, and $\mathrm{EVH}$ was associated with a higher rate of repeat revascularization.

Considering that no specific guidelines (except for the 2005 International Society for Minimally Invasive Cardiac Surgery's consensus statement ${ }^{22}$ ) are currently available to

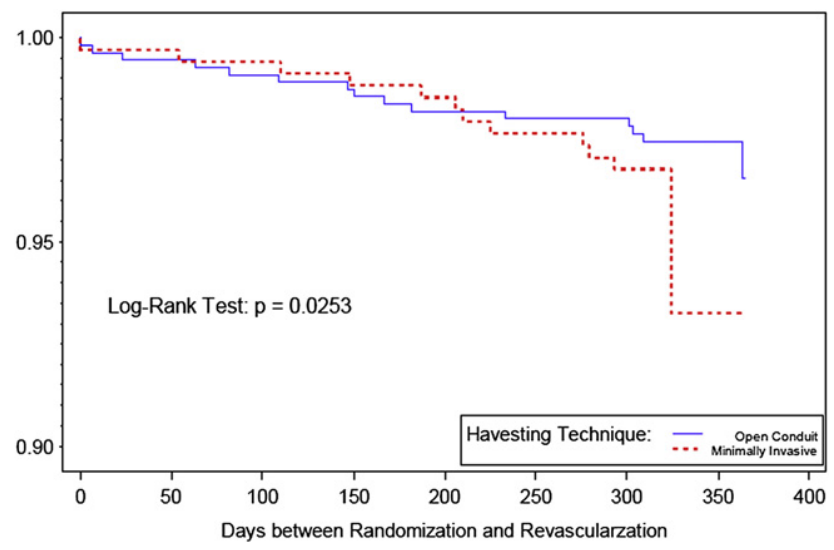

FIGURE 2. Kaplan-Meier estimate of time to repeat revascularization $(\mathrm{n}=894)$. assist the clinician in decision making regarding the SVG harvest technique, the risks and benefits of EVH relative to $\mathrm{OVH}$ need to be carefully addressed with the patient and referring cardiologist on a case-by-case basis until more definitive evidence becomes available. In addition, further research may be required to find and support methods of preventing graft failure, possibly including implementation of a more intense perioperative antithrombotic regimen when using EVH. ${ }^{23,24}$ In light of the recent meta-analysis and these ROOBY Trial findings, a large, prospective, randomized study now appears warranted.

We are indebted to Janet H. Baltz, RN, Annette Wiseman, Jennifer Carrick, RN, MS, CCRC, Jennifer M. Gabany, MSN, CRNP-C, CCRC, and Timothy Miller, PA-C, for their invaluable contributions to this study.

\section{References}

1. Lumsden AB, Eaves FF 3rd, Ofenloch JC, Jordan WD. Subcutaneous, videoassisted saphenous vein harvest: report of the first 30 cases. Cardiovasc Surg. 1996;4:771-6.

2. Brown ML, Sundt TM. Surgical coronary artery revascularization. In: Yusuf S, Cairns JA, Camm AJ, Fallen EL, Gersh BJ, eds. Evidence-based cardiology. 3rd ed. Hoboken (NJ): Wiley-Blackwell; 2010. p. 379-91.

3. Andreasen JJ, Nekrasas V, Dethlefsen C. Endoscopic vs open saphenous vein harvest for coronary artery bypass grafting: a prospective randomized trial. Eur J Cardiothorac Surg. 2008;34:384-9.

4. Puskas JD, Wright CE, Miller PK, Anderson TE, Gott JP, Brown WM 3rd, et al. A randomized trial of endoscopic versus open saphenous vein harvest in coronary bypass surgery. Ann Thorac Surg. 1999;68:1509-12.

5. Kiaii B, Moon BC, Massel D, Langlois Y, Austin TW, Willoughby A, et al. A prospective randomized trial of endoscopic versus conventional harvesting of the saphenous vein in coronary artery bypass surgery. $J$ Thorac Cardiovasc Surg. 2002;123:204-12. Erratum in: J Thorac Cardiovasc Surg. 2002;123:1224.

6. Markar SR, Kutty R, Edmonds L, Sadat U, Nair S. A meta-analysis of minimally invasive versus traditional open vein harvest for coronary artery bypass graft surgery. Interact Cardiovascular Thorac Surg. 2010;10:266-70.

7. Cheng DC, Martin J, Ferdinand FD, Puskas JD, Diegler A, Allen KB. Endoscopic vein-graft harvesting: balancing the risk and benefit. Innovations. 2010; 5:70-3.

8. Magee MJ, Alexander JH, Hafley G, Ferguson TB Jr, Gibson CM, Harrington RA, et al. Coronary artery bypass graft failure after on-pump and off-pump coronary artery bypass: findings from PREVENT IV. Ann Thorac Surg. 2008;85:494-500.

9. Lopes RD, Hafley GE, Allen KB, Ferguson TB, Peterson ED, Harrington RA, et al. Endoscopic versus open vein-graft harvesting in coronary-artery bypass surgery. N Engl J Med. 2009;361:235-44.

10. Connolly MW, Poston RS. Endoscopic versus open vein-graft harvesting. $N$ Engl J Med. 2009;361:1907-8.

11. Aranki SF, Shopnick B. Endoscopic versus open vein-graft harvesting. $N$ Engl J Med. 2009;361:1907.

12. Patel NP, Angelini GD. Open or endoscopic vein graft harvesting-this is the question! Nat Rev Cardiol. 2009;6:738-40.

13. Shroyer AL, Grover FL, Hattler B, Collins JF, McDonald GO, Kozora E, et al. On-pump versus off-pump coronary-artery bypass surgery. $N$ Engl J Med. 2009;361:1827-37. 
14. Novitzky D, Shroyer AL, Collins JF, McDonald GO, Lucke J, Hattler B, et al. A study design to assess the safety and efficacy of on-pump versus off-pump coronary bypass grafting: the ROOBY trial. Clin Trials. 2007;4:81-91.

15. FitzGibbon GM, Burton JR, Leach AJ. Coronary bypass graft fate: angiographic grading of 1400 consecutive grafts early after operation and of 1132 after one year. Circulation. 1978;57:1070-4.

16. Sabik JF 3rd, Lytle BW, Blackstone EH, Houghtaling PL, Cosgrove DM. Comparison of saphenous vein and internal thoracic artery graft patency by coronary system. Ann Thorac Surg. 2005;79:544-51.

17. Rousou LJ, Taylor KB, Lu XG, Healey N, Crittenden MD, Khuri SF, et al. Saphenous vein conduits harvested by endoscopic technique exhibit structural and functional damage. Ann Thorac Surg. 2009;87:62-70.

18. Widimsky P, Straka Z, Stros P, Jirasek K, Dvorak J, Votava J, et al. One-year coronary bypass graft patency: a randomized comparison between off-pump and on-pump surgery angiographic results of the PRAGUE-4 trial. Circulation. 2004;110:3418-23.

19. Perrault LP, Jeanmart H, Bilodeau L, Lespérance J, Tanguay JF, Bouchard D, et al. Early quantitative coronary angiography of saphenous vein grafts for coronary artery bypass grafting harvested by means of open versus endoscopic saphenectomy: a prospective randomized trial. J Thorac Cardiovasc Surg. 2004; 127:1402-7.

20. Yun KL, Wu Y, Aharonian V, Mansukhani P, Pfeffer TA, Sintek CF, et al. Randomized trial of endoscopic versus open vein harvest for coronary artery bypass grafting: six-month patency rates. J Thorac Cardiovasc Surg. 2005; 129:496-503.

21. Puskas JD, Halkos ME, Balkhy H, Caskey M, Connolly M, Crouch J, et al. Evaluation of the PAS-Port proximal anastomotic system in coronary artery bypass surgery (the EPIC trial). J Thorac Cardiovasc Surg. 2009;138:125-32.

22. Allen K, Cheng D, Cohn W, Connolly M, Edgerton J, Falk V, et al. Endoscopic vascular harvest in coronary artery bypass grafting surgery: a consensus statement of the international society of minimally invasive cardiothoracic surgery (ISMICS) 2005. Innovations. 2005;1:51-60.

23. Brown EN, Kon ZN, Tran R, Burris NS, Gu J, Laird P, et al. Strategies to reduce intraluminal clot formation in endoscopically harvested saphenous veins. J Thorac Cardiovasc Surg. 2007;134:1259-65.

24. Bhatt DL. Role of antiplatelet therapy across the spectrum of patients with coronary artery disease. Am J Cardiol. 2009;103(3 Suppl):11A-9.

\section{Discussion}

Dr Carlos A. Mestres (Barcelona, Spain). I have to disclose that I am on the advisory board for Novartis Pharma and I have done temporary consulting work for Edwards Lifesciences and St Jude Medical in Europe.

Dr Zenati, as you know very well, the ROOBY Trial concluded that at 1-year follow-up, patients undergoing off-pump CABG had worse composite outcomes and poorer graft patencies than those undergoing on-pump CABG. More interestingly, in your study, when EVH was compared with OVH, 1-year patency was significantly lower and revascularization rate significantly higher for EVH.

Despite the proven advantages of EVH relative to OVH in terms of wound complications and cosmesis, your data incorporated information showing that 1-year graft patency might be compromised with endoscopic harvesting. Previous studies that included smaller populations and angiography performed within the first 6 postoperative months did not show differences in patency.

Your data support a nonrandomized study showing worse outcomes with EVH, and generally speaking it appears that SVG failure is higher with EVH, as also shown in a recent meta-analysis. In addition, your patient population seems to be a good representation of "real life," at least on the male side.

Reduced graft patency with EVH could be related to intraoperative trauma, which is inherent in the technique. There is also information suggesting that EVH may impair endothelial function.

The eventual implications of your study, in combination with the available information on graft patency related to the harvesting technique, could have potential impact on clinical practice and perhaps relegate EVH and its association with off-pump surgery to the suboptimal side in terms of long-term results. Your study appears to be sufficiently powered when compared to previous studies to draw meaningful conclusions, despite a number of limitations that you clearly addressed in your presentation and also in your manuscript.

Finally, before proceeding with my questions, I obviously have to thank you for this significant and controversial piece of information and the opportunity for advanced manuscript review and the Association for the privilege of the discussion.

I have basically 3 questions for you. First, do you have information about which were the territories with the highest occlusion rate? If so, did that have a clinical impact on your patients?

Dr Zenati. Thank you, Dr Mestres, for your kind comments and your question. Regarding the distribution of the SVGs and relative patency as related to the harvesting technique, the right coronary distribution, interestingly enough, did not show any difference in terms of patency whether the conduit harvest was endoscopic or open. The patency was $87.5 \%$ for EVH and $85.6 \%$ for OVH. The difference was not statistically significant. When we looked at the diagonal and obtuse marginal coronary arteries, the patency was significantly higher for both targets when the harvest was open. Patency was $69.7 \%$ in the obtuse marginal branch with EVH, versus $82.9 \%$ with OVH.

Dr Mestres. Second, considering that a limitation is that you did not randomly assign the harvesting technique, I find some differences with especially the PREVENT trial, because that was organized in the early phases of the learning curve with harvesting techniques, whereas your trial was, of course, more recent in terms of chronology. So, would you suggest that going through the learning curve in harvesting on an endoscopic basis is not a major problem in this trial?

Dr Zenati. You raise the issue of experience with harvesting, and our trial does cover a more recent period than did the PREVENT subanalysis. As we saw, the penetration of EVH technique in the United States at the beginning of the ROOBY Trial in 2002 was probably less than $10 \%$, rising almost exponentially to about $76 \%$ in 2008 . We were not able to account for the effect of the level of experience or the volume of procedures performed by the practitioner who harvested the SVGs, however, because these data were not collected.

Dr Mestres. Third, what is your current antithrombotic therapy? Also, assuming that EVH represents basically induced endothelial trauma, do you think that a more intense antithrombotic regimen would be of any help?

Dr Zenati. You raise a very important point about the perioperative antithrombotic approach in relationship to the potential for increased endothelial damage during the harvest. Aspirin use was ubiquitous in this study, and about $30 \%$ of patients also received clopidogrel. The CASCADE trial was just presented last fall at the American Heart Association, however, and its results were negative in terms of the ability of the dual antiplatelet to improve graft patency. This is something we need to reassess, and potentially we need to include consideration of pharmacogenomics 
to take into account low metabolizers when prescribing clopidogrel. On top of that, there is the issue of heparin at the beginning of harvest, which has been shown in some series to affect graft failure, but these data were not collected in this study and should be in the future.

Dr Mestres. Thank you very much for this important contribution.

Dr Omar Lattouf (Atlanta, Ga). Dr Zenati, thank you for your presentation. The points that you raise have significant medicolegal impact for those of us who use EVH. First, in defense of EVH, I have to say that the data that you have presented did not take into account any technical issues that were encountered at the time of vein harvest either way and were not recorded. Second, we do not know the starting point for the conduit patency documented by angiography, coronary ultrasonography, or flow patencies. If you do have those data, can you share them with us, please?

Dr Zenati. Thank you. Regarding the technical issue, as I indicated, a limitation of the study is that device-related or experience issues may have affected outcomes, and these data unfortunately were not collected, so I cannot comment on that. I believe that future studies should consider device-related variables very seriously, because the EVH technique is improving and evolving.

With respect to the timing of the failure of the grafts, most (more than 90\%) were protocol angiographies. So the graft could have been closed at an earlier stage, and that is unfortunately difficult to evaluate and comment on. 